\title{
LA TRAGEDIA DE LA PROPIEDAD INTELECTUAL (I): UNA ERRÓNEA ASIGNACIÓN DE TITULARIDADES
}

\author{
HUARAG GUERRERO, Enrico ${ }^{147}$
}

SUMARIO: 1. La asignación de titularidades sobre bienes. - 2. La ausencia de titularidades: la tragedia de los comunes. - 3. Rivalidad en el consumo y costos en la definición del tratamiento de los bienes. -4 . De la ausencia al exeso de titularidades: la tragedia de los anticomunes. -5 . El tratamiento de la propiedad intelectual. - 6. ¿Qué papel debe jugar la propiedad intelectual?

En 2004, Google Inc. inició un proyecto de escaneado de libros que buscaba convertirse en la mayor biblioteca mundial. Sin embargo, problemas con los derechos de autor de las denominadas "obras huérfanas" se convirtieron en verdaderos obstáculos que terminaron por paralizar el proyecto y convertirlo en el actual Google Books.

En los últimos años hemos venido siendo testigos de una verdadera guerra comercial a escala mundial librada entre las más grandes compañías tecnológicas: Google, Apple, Samsung, Microsoft, Nokia, entre otras. Las armas utilizadas son las patentes. Millones de dólares que podrían ser invertidos en investigación e innovación son destinados a comprar y registrar "patentes defensivas" que protejan a las compañías frente a futuras demandas de sus competidoras. De esta forma, muchos productos dejan de salir al mercado ante la amenaza de un proceso por infracción de patentes y muchas tecnologías no se desarrollan por destinar sus presupuestos a patentes defensivas. Los perdedores, como siempre, los consumidores.

Cada año, miles de millones de dólares son utilizados en la lucha contra la piratería, pero los niveles de ésta no han descendido de manera significativa. Por otro lado, vemos con preocupación la manera en la que ganan terreno determinadas iniciativas legislativas que recortan derechos fundamentales de los ciudadanos bajo la consigna de la protección de la propiedad intelectual. Así, la lucha contra la piratería de contenidos se convierte en el pretexto para un mayor control por parte de los gobiernos sobre los medios de información digital, la censura, la invasión de la privacidad, y la restricción del conocimiento libre. Y el fenómeno se reproduce en muchos países, como podemos ver con la Ley Hadopi en Francia, la Ley Sinde en España, la Stop Online Piracy Act (SOPA) en Estados Unidos, o en acuerdos internacionales, como el Anti-Counterfeiting Trade Agreement (ACTA).

$147 \quad$ El autor es Máster en Derecho Privado por la Universidad Carlos III de Madrid. En la actualidad se desempeña como profesor de los cursos de Derecho y Economía y Derecho Administrativo de la Facultad de Derecho y Ciencias Políticas de la Universidad Ricardo Palma.

El presente trabajo forma parte de una serie de tres artículos en los que se expondrá la problemática actual de la propiedad intelectual, buscando propuestas de solución enfocadas a nuevas formas de tratamiento de la misma y la generación de nuevos modelos de negocios. 
Pero, ¿cómo hemos llegado a esta situación? ¿En qué momento las patentes dejaron de incentivar la innovación para convertirse en verdaderos obstáculos para la misma? ¿En qué momento los derechos de autor ${ }^{148}$ dejaron de incentivar la creación y se transformaron en una amenaza a las libertades de los individuos?

A nuestro entender, estos problemas tienen su origen en la misma concepción de los derechos de propiedad intelectual. En este primer este primer artículo veremos que el meollo de la cuestión se encuentra en una incorrecta asignación de titularidades, que tuvo como consecuencia la construcción de un sistema que aparentemente incentivaba la innovación y la creación, pero cuya ineficiencia recién pudo ser apreciada con el auge de nuevas tecnologías como la Internet.

\section{La asignación de titularidades sobre bienes}

La primera tarea que debe asumir cualquier sistema jurídico es resolver el problema de la asignación de titularidades de los derechos. Esto es, el Estado tiene como cuestión fundamental decidir a quién le asigna tal o cual derecho:

"El primer asunto que cualquier sistema jurídico debe encarar es aquel que llamamos el problema de la "titularidad de los derechos". Cada vez que un Estado enfrenta un conflicto de intereses entre dos o más personas, o entre dos o más grupos de personas, debe decidir a cuál de las partes favorecer. En ausencia de esta decisión, el acceso a los bienes, servicios y a la vida misma será decidido sobre la base de que "el poder hace el Derecho” (might makes right), de modo que quien sea más fuerte o más hábil ganará. De aquí que la cuestión fundamental que hace el Derecho es decidir cuál de las partes en conflicto tendrá el derecho a prevalecer" ${ }^{149}$.

Uno de los pilares del funcionamiento del sistema económico es el derecho de propiedad. Como señala Posner, la protección legal de los derechos de propiedad crea incentivos para el uso eficiente de los recursos ${ }^{150}$. Por ello, resulta de capital importancia asignar correctamente las titularidades y derechos sobre los bienes, generando de esta los incentivos necesarios para su uso adecuado y su provisión suficiente ${ }^{151}$.

148 A lo largo de este trabajo utilizaré la expresión "derechos de autor" para referirme específicamente al copyright. Tengo que aclarar que el concepto de derechos de autor incluye la noción anglosajona del copyright en sentido estricto (el derecho de controlar la reproducción de la obra), y además da a entender que el concepto incluye también una gama más amplia de derechos adicionales. Entre estos se incluye los denominados "derechos morales" del autor (derechos de paternidad, protecciones contra el plagio, integridad de la obra, etc.) que son inalienables y perpetuos, y los que comprende el copyright en sentido amplio, que vendrán a ser los derechos patrimoniales del autor.

149 CALABRESI, Guido y MELAMED, A. Douglas. "Reglas de propiedad, reglas de responsabilidad y de inalienabilidad: una vista de la catedral”. En: Estudios públicos. No.63. Santiago de Chile: Centro de Estudios Públicos, 1996, p. 349.

150 Posner, Richard. El Análisis Económico del Derecho. México: Fondo de Cultura Económica, 2000, p. 38.

151 Este tipo de asignación de titularidad sobre bienes es definida por Calabresi y Malamed como una regla de propiedad. Al respecto, señalan que "un derecho es protegido por una regla de propiedad en la medida en que quien desea quitarle el derecho a su titular debe comprárselo en una transacción voluntaria, en la que el valor del derecho es aceptado por quien lo enajena. Esta es la forma de titularidad que da pie a la menor cantidad de intervención estatal: una vez decidida la titularidad original del derecho, el Estado no intenta decidir su valor. Se permite que cada parte manifieste cuánto vale el derecho para sí misma, y otorga al vendedor un veto si el comprador no ofrece lo suficiente. Las reglas de propiedad implican una decisión colectiva en cuanto a quién habrá de concedérsele un derecho inicial, pero no en cuanto al valor de ese derecho". Ver: CALABRESI, Guido y MELAMED, A. Douglas. Óp. cit., p. 351. 
Desde un punto de vista económico, resulta necesario definir y proteger derechos de propiedad privada debido a que los bienes son escasos. En ese sentido, no tiene mucho sentido delimitar derechos de propiedad sobre bienes cuando éstos existen en abundancia. Así, por ejemplo, no establecemos derechos de propiedad sobre el aire, sobre la luz solar, u otros, toda vez que -al menos de momento- no existe escasez de los mismos ${ }^{152}$.

Sin embargo, cuando los bienes son escasos, la propiedad comunal de los mismos no permite que éstos sean utilizados de manera eficiente. La propiedad privada, en cambio, garantiza que los bienes escasos serán usados de la forma más eficiente y productiva. Por esta razón, el Sistema Jurídico crea una serie de derechos denominados "reales" que van a ser oponibles erga omnes.

"La propiedad privada sobre ellos permite que se genere un mayor número de bienes que por naturaleza son escasos.

En primer lugar, se evita la sobreexplotación del bien. Cuando un bien privado es de acceso abierto al público, los individuos lo utilizan sin tener en cuenta los costos que su aprovechamiento impone en el resto. Básicamente, se explota el bien de manera indiscriminada y desordenada, lo cual suele determinar su agotamiento. Al brindarle a un individuo la posibilidad de excluir al resto del aprovechamiento del bien -al crear un derecho de propiedad privada-permitimos que él asuma todos los costos y beneficios de su explotación" 153 .

Profundicemos un poco sobre esta idea. Si tenemos un campo y un individuo lo trabaja, compra fertilizantes y semillas, construye canales de regadío y hasta un espantapájaros, pero cuando va a recoger la cosecha se encuentra con que sus vecinos son quienes se están beneficiando con los frutos, recogiendo lo que él ha sembrado, sin que pueda hacer nada para evitarlo, carecerá de los incentivos necesarios para repetir esta conducta y probablemente abandone la agricultura y la sociedad cambiará a los métodos de subsistencia que involucran una inversión preparatoria menor ${ }^{154}$. En este tipo de sistemas, se carece de incentivos para hacer productivas las tierras. Si el beneficio de nuestras actividades se traslada a terceros en forma de externalidades positivas, sin que podamos disfrutarlas, no existen incentivos para desarrollar la actividad. Para que alguien invierta capital y trabajo en hacer productivo el campo, deberá tener algún tipo de derecho que le permita excluir a los demás, de manera que pueda aprovechar la inversión que realiza. Y es la propiedad privada ese derecho, el que le permite excluir a los demás, y al hacerlo, crea el incentivo necesario para que haya inversión en volver productivo el campo. En ese sentido, como señala POSNER, "si todo pedazo de tierra es propiedad de alguien -si siempre hay alguien que puede excluir a todos los demás del acceso a cualquier área dada-, los individuos tratarán de maximizar el valor de la tierra mediante el cultivo u otras mejoras"." 155

152 Posner ejemplifica la relación entre escasez y propiedad privada con la diferencia entre el tratamiento legal del agua entre el este y el oeste de los Estados Unidos de América. Así, en los Estados del este, donde el agua es abundante, los derechos sobre el agua tienden a ser comunales. En cambio, en el oeste, donde el agua es escasa, pueden obtenerse derechos exclusivos sobre esta mediante la apropiación. Ver: POSNER, Richard. Óp. cit., p. 42.

PASQUEL RODRIGUEZ, Enrique, Una visión crítica de la propiedad intelectual: por qué eliminar las patentes, los derechos de autor y el subsidio estatal a la producción de información. En: Revista Economía y Derecho. 2004 Vol. I, No 3. Lima: Sociedad de Economía y Derecho UPC, 2004, p. 69

154 POSNER, Richard. Óp. cit., p. 38

155 Idem. 


\section{La ausencia de titularidades: la tragedia de los comunes}

Cuando no se asignan derechos de propiedad sobre bienes escasos, se produce lo que HARDIN ha denominado la tragedia de los comunes. Imaginemos un pastizal de acceso abierto a todos ${ }^{156}$. Cada pastor pues recibe todos los beneficios de la venta de los animales de su rebaño. Sin embargo, la utilidad negativa producida por el sobrepastoreo son compartidos por todos los pastores que utilizan el pastizal, por lo que realmente asume solo una fracción de este costo.

En este contexto, el pastor racional asume que la única decisión sensata para él es añadir más animales para que pasten, conclusión a que también llegan cada uno y todos los pastores que comparten el recurso común, agotando el recurso, configurándose así los elementos de la tragedia:

"Adding together the component partial utilities, the rational herdsman concludes that the only sensible course for him to pursue is to add another animal to his herd. And another; and another... But this is the conclusion reached by each and every rational herdsman sharing a commons. Therein is the tragedy. Each man is locked into a system that compels him to increase his herd without limit--in a world that is limited. Ruin is the destination toward which all men rush, each pursuing his own best interest in a society that believes in the freedom of the commons. Freedom in a commons brings ruin to all." 157

Como vemos en el ejemplo brindado por HARDIN, los usuarios de bienes comunes solo tienen en cuenta el beneficio producido de la explotación, y no el costo, que termina convertido en una externalidad negativa a repartir entre todo. Este sistema incentivará a incrementar la actividad de explotación del recurso. Al no poder excluir a los demás, nadie que se ocupa de invertir en la preservación del bien común, toda vez que el gasto sería asumido por uno, pero el beneficio dividido entre todos. El resultado final será el agotamiento del recurso por sobreexplotación y la ruina de quienes se dedican a dicha actividad. Y es que un sistema de propiedad común, igual que un sistema sin propiedad, lleva a que se utilice como infinito un bien que en realidad es escaso. La conducta generada por estos sistemas no se adecúa al nivel de escasez del bien ${ }^{158}$.

Son estas dos características, la escasez de los bienes protegidos, y la posibilidad de excluir a los otros de su consumo, las que determinarán el sistema de propiedad más eficiente en cada caso. En ese sentido, la propiedad privada clásica se justifica por la escasez de los

156 En este punto, conviene precisar que cuando hablamos de bienes comunes nos referimos a bienes de acceso abierto a todos. Existen casos en que el acceso a un bien es relativamente libre, pero se encuentra controlado y regulado por una comunidad. Conforme demuestran los trabajos de Elionor Ostrom (Premio Nobel de Economía 2009), en el caso de bienes administrados por comunidades, en el que existan mecanismos que permiten a esa comunidad excluir del uso a los no miembros, los bienes comunes pueden ser administrados de forma eficiente, aunque bajo determinadas condiciones que permitan un enforcement efectivo de las decisiones de la comunidad. En cualquier caso, tanto la facultad de excluir a los demás como la capacidad de llegar a acuerdos y hacerlos efectivos (esto es, bajos costos de transacción), son elementos claves en el funcionamiento eficiente del sistema.

157 HARDIN, Garret, The Tragedy of the Commons. En: Science. 13 diciembre 1968. Vol. 162. N ${ }^{\circ}$ 3859. Stanford: American Association for the Advancement of Science, 1968, p. 1244.

158 Bullard GonZalez, Alfredo. Derecho y Economía: Análisis económico de las instituciones legales. 2da. Ed. Lima: Palestra Editores, 2006. pp. 153-154. 
bienes, de manera que el sistema de propiedad sirve para la correcta asignación de los mismos, creando incentivos para la conservación del bien objeto de la titularidad y su utilización eficiente y productiva.

\section{Rivalidad en el consumo y costos de exclusión en la definición del tratamiento de los bienes}

Debemos tener en cuenta que no a todos los bienes se les pude asignar de manera eficiente el tratamiento de propiedad privada. Hay casos en los cuales la eficiencia económica aconseja tratamientos distintos. Así, estudiaremos dos atributos que diferencian a los bienes objeto de propiedad privada de aquellos que tienen un tratamiento de bien público: la rivalidad en el consumo y los costos de exclusión.

La rivalidad en el consumo puede ser definida como la imposibilidad de que un bien pueda ser utilizado dos personas para dos fines distintos de manera simultánea. Se trata de un concepto económico: un acto de consumo excluye otro acto de consumo. Por ejemplo, un coche no puede ser utilizado para ir a dos lugares distintos al mismo tiempo, y si una persona se come una manzana, otras personas no podrán comerla. ${ }^{159}$ Lo que consume uno, lo pierde el otro. La propiedad que disfruta uno, la pierde el rival.

BULLARD cita como ejemplo clásico de consumo no rival el de la seguridad pública. El solo hecho de colocar un policía en una esquina, dando seguridad a los vecinos de una calle, no excluye la seguridad a los demás. La sola presencia del policía desincentiva que se roben los hogares de toda la calle y a las personas que por ella transitan. En ese sentido, darle seguridad a un vecino significa que los demás quedarán incluidos en la misma seguridad ${ }^{160}$. En términos económicos, se ha generado una externalidad positiva. El hecho de que un vecino disfrute de seguridad no significa que los otros no gocen del mismo tipo de seguridad de manera simultánea. En ese sentido, los consumos no son rivales.

Por su parte, los costos de exclusión pueden ser definidos como aquellos en los que debe incurrir un propietario para excluir a los terceros del uso de un bien. En algunos casos, excluir a los demás del uso de un bien puede ser muy costoso, como en el caso del aire. Normalmente, en estos casos el sistema jurídico opta por considerar a este tipo de bienes como de uso público, pues nos no hay rivalidad en el consumo ${ }^{161}$.

Los costos de exclusión, en cambio, suelen ser bajos en el caso de bienes que pueden ser identificados o que tienen un límite físico identificable. Si quiero excluir a los demás del uso de una parcela, basta con que construya una cerca. Si quiero excluir a los demás del uso de un reloj de pulsera, basta que lo mantenga dentro de mi esfera de dominio. Del mismo modo, crear mecanismos jurídicos de exclusión en estos casos es relativamente sencillo:

"Nuevamente, los costos de exclusión suelen ser bajos en los típicos bienes sujetos a la propiedad clásica, como una casa o un libro. Lo que ocurre es que, en bienes que pueden ser identificados y que tienen un límite físico identificable, crear

159 CoOTER, Robert; Ulen, Thomas. Derecho y Economía. México: Fondo de Cultura Económica, 1998, p. 144.

$160 \quad$ Bullard GONZALEZ, Alfredo. Óp. cit., p. 157.

161 COOTER, Robert y ULEN, Thomas. Loc. cit. 
mecanismos de exclusión como registros, acciones judiciales y defensa posesoria, es relativamente sencillo". 162

Por regla general, se establecen derechos de propiedad privada sobre los bienes que presentan rivalidad en el consumo y bajos costos de exclusión. De esta forma, se asignan derechos de propiedad privada, sobre las parcelas, sobre las casas, los automóviles, los barcos, etc. Por otro lado, si se presenta rivalidad en el consumo, y los costos de exclusión son altos, el bien pasa a ser de uso público, como en el caso del aire que respiramos, la seguridad pública, etc.

Hay que señalar que las características de rivalidad en el consumo y bajos costos de exclusión pueden no presentarse juntas. En el caso de los cardúmenes, se presenta rivalidad en el consumo, pues si uno se come un pez, el mismo no puede ser comido por otro. Pero debido a su naturaleza móvil en mares con diferentes soberanías y legislaciones, son considerados bienes privados imperfectos. Toda vez que los cardúmenes se mueven por el océano, identificar a quién pertenece un pez específico es muy costoso, el resultado es que, en principio, no existe propiedad sobre los cardúmenes. Y es que la propiedad, en este caso, resultaría ineficiente. En consecuencia, para evitar problemas típicos de los bienes de uso público como la sobreexplotación, se utilizan mecanismos alternativos a la propiedad, pero más eficientes, como vedas o la asignación de cuotas de pesca.

BULLARD también ilustra casos en que no existe rivalidad en el consumo, pero los costos de exclusión son relativamente bajos. Pensemos en el caso de las autovías. El hecho de que la utilice un automóvil no significa que otro no pueda utilizarla de manera simultánea, y así, miles de automóviles diariamente. Pero los costos de exclusión son en este caso tan reducidos como construir una caseta de peaje, con la que podríamos excluir a un número determinado de automóviles para evitar la congestión de la misma. Nos encontramos ante lo que se conoce como un bien público imperfecto.

También podemos encontrarnos con casos en los que no se cumplen los requisitos de rivalidad en el consumo y de bajos costos de exclusión. En estos casos, al no existir rivalidad en el consumo y ser los costos de exclusión muy altos (como en el caso del aire o de la seguridad pública), se asigna a los bienes el carácter de bienes públicos.

Sobre estas consideraciones, BULLARD propone el siguiente esquema de asignación de derechos de propiedad, en función a la existencia de rivalidad en el consumo y la magnitud de los costos de exclusión ${ }^{163}$ :

\begin{tabular}{|c|c|c|c|c|}
\hline \multirow{2}{*}{ R } & \multicolumn{3}{|c|}{ COSTOS DE EXCLUSION } \\
\cline { 2 - 4 } I & & Bajos & Altos \\
\cline { 2 - 4 } V & & Propiedad & Bienes Privados \\
A & SI & Privada & Imperfectos \\
L & & & \\
I & & & Bienes Públicos & Bienes Públicos \\
D & & \multirow{2}{*}{ Imperfectos } & \\
D & NO & Bing \\
\end{tabular}


Como podemos ver, es la existencia de rivalidad en el consumo y la magnitud de los costos de exclusión la que determinará el tratamiento adecuado de los bienes y la forma de asignar de manera económicamente eficiente la titularidad de derechos sobre los mismos. De acuerdo a COOTER y ULEN, la ley puede lograr la asignación eficiente de los bienes privados reduciendo los costos de negociación mediante la asignación de derechos de propiedad claros y simples. Así, el dueño de un bien privado tendrá la facultad de excluir a otros del uso de los bienes, lo que canaliza el uso de estos hacia el intercambio voluntario, lo que promueve el uso eficiente. Por el contrario, los mercados tenderán a proveer pocos bienes públicos, pues el proveedor privado no puede excluir a los free riders (aquellos que no pagan su consumo de un bien público) del uso de dicho bien ${ }^{164}$.

\section{De la ausencia al exceso de titularidades: la tragedia de los anticomunes}

La tragedia de los comunes es solo uno de los supuestos que pueden presentarse por la existencia de derechos de propiedad mal definidos. Esta se produce por la existencia de varias personas con titularidades conjuntas de derechos de uso de un determinado bien, sobre el cual ninguna de ellas tiene el derecho de excluir a los demás. Sin embargo, puede presentarse un supuesto opuesto de derechos de propiedad mal definidos: la existencia de varios titulares que poseen derechos conjuntos de exclusión sobre un determinado bien, a pesar de lo cual ninguno de ellos ostenta derechos exclusivos de uso ${ }^{165}$. Es lo que HeLLER denominó tragedia de los anticomunes:

"By contrast, a resource is prone to underuse in a "tragedy of the anticommons" when multiple owners each have a right to exclude others from a scarce re-source and no one has an effective privilege of use. In theory, in a world of costless transactions, people could always avoid commons or anticommons tragedies by trading their rights. In practice, however, avoiding tragedy requires overcoming transaction costs, strategic behaviors, and cognitive biases of participants, with success more likely within closeknit communities than among hostile strangers. Once an anticommons (sic) emerges, collecting rights into usable private property is often brutal and slow. ",166

Como podemos apreciar, en el caso de los anticomunes se presenta como presupuesto la existencia de pluralidad de titularidades sobre un mismo bien, lo que dificulta poder llegar a acuerdos sobre la correcta administración del mismo. En la medida en que cada uno de los titulares ostenta derechos de exclusión sobre los otros titulares (normalmente expresados como derechos de veto), se elevan los costos de transacción en la toma de las decisiones necesarias para la administración eficiente del bien, el mismo que termina siendo desperdiciado por subexplotación.

Existen varios ejemplos en los que se presentan situaciones de anticomunes. En Moscú, en plena transición de la economía comunista a la economía de mercado, era común observar que mientras el comercio florecía en los pequeños kioscos de las calles, pero las transacciones no se llevaban a cabo dentro de los negocios, que permanecían vacíos. A pesar

165 COOTER, Robert y ULEN, Thomas. Óp. cit., pp. 144-145.

HELler, Michael, The Tragedy of the Anticommons: Property in the transition from Marx to Markets. En: Harvard Law Review, enero 1998, Vol. 111, No 3. Harvard: The Harvard Law Review Association, 1998, p. 668.

166 Heller, Michael y EISENBERG, Rebecca. Can Patents Deter Innovation? The Anticommons in Biomedical Research. En: Science. 1 mayo 1998. Vol. 280. No 5364. Stanford: American Association for the Advancement of Science, 1998, p. 698. 
que de que los propietarios de los kioscos estaban deseosos de alquilar los negocios, al tener estos distintas titularidades (derechos de propiedad incompletos), por lo que permanecían como un valioso recurso inexplotado ${ }^{167}$.

Otro ejemplo lo tenemos en el campo de la investigación biomédica, tan necesaria para la generación de productos que salven vidas humanas, en el que cada nuevo avance requiere de la utilización de descubrimientos científicos previos. Normalmente estos descubrimientos han sido materia de patentes, por lo que el avance en biomedicina requiere el pago de licencias a quienes detentan las respectivas patentes, quienes además tienen la potestad de bloquear el acceso a las mismas. La excesiva proliferación de derechos de propiedad intelectual (en este caso, patentes) muy restrictivos eleva innecesariamente los costos de acceso, conduciendo paradójicamente a un menor desarrollo de productos necesarios para preservar la salud ${ }^{168}$ y salvar vidas, con el consiguiente perjuicio para la Sociedad:

"Not only do research labs lose potential profits, but families lose loved ones and communities lose friends and neighbors. Research scientists have whispered to me about other potential cures blocked by a multiplicity of patent owners. These missing drugs are a silent tragedy. Millions have suffered and will continue to suffer or die from diseases that could have been treated or prevented, but no one protests. Where do you go to complain about lifesaving drugs that could exist-should exist-but don't? How do you mobilize public out-rage about the gridlock economy in drug innovation?

Un ejemplo local de la tragedia de los anticomunes lo tenemos en el Centro Comercial Camino Real, con locales vacíos, nula inversión publicitaria y con un deterioro creciente e irremediable. En este caso - a diferencia de otros centros comerciales de Lima, en los cuales un solo dueño o una administración centralizada que alquila tiendas- se vendió cada tienda a distintos propietarios. Sin una administración centralizada, los propietarios no logran llegar a acuerdos para promover, administrar e invertir en la conservación del Centro Comercial. Los intentos de venta o de inversión en mejoras fracasan porque existen pequeños grupos que se niegan a hacerlo ${ }^{170}$.

Los ejemplos anteriores nos demuestran que una mala asignación de titularidades a través de derechos de propiedad fragmentados o de regulaciones restrictivas incrementan los costos de transacción y dificultan llegar a acuerdos para una administración eficiente de los bienes, provocando una tragedia. Como señalara HELLER, si bien la propiedad privada por lo general aumenta la riqueza, un exceso de propiedad tiene el efecto contrario: destruye los mercados, detiene la innovación, y cuesta vidas ${ }^{171}$.

167 HELLER, Michael. The Tragedy of the Anticommons: Property in the transition from Marx to Markets, pp. 622-623.

HELLER, Michael y EISENBERG, Rebecca. Can Patents Deter Innovation? The Anticommons in Biomedical Research, p. 701.

169 HELLER, Michael. The Gridlock Economy: How Too Much Ownership Wrecks Markets, Stops Innovation, and Costs Lives. New York: Basic Books, 2008, p. 6.

BUllard GONZÁlEZ, Alfredo. "Bagua y la tragedia de los anticomunes". En: Prohibido Prohibir. Blog de semanaeconomica.com. Post de 24 de junio de 2009. Disponible en formato electrónico en: [http://blogs.semanaeconomica.com/blogs/prohibido-prohibir/posts/bagua-y-la-tragedia-de-los-nticomunes]. 
Esto nos demuestra que nos encontramos ante un espectro de asignación de titularidades, a cuyos extremos nos encontramos con el acceso abierto a todos, en el que exclusión resulta imposible (esto es, el supuesto de la tragedia de los comunes), y la exclusión completa, en el que las titularidades sobre el bien se encuentran tan dispensas que resulta imposible sacar provecho del mismo (esto es, una tragedia de los anticomunes) ${ }^{172}$.

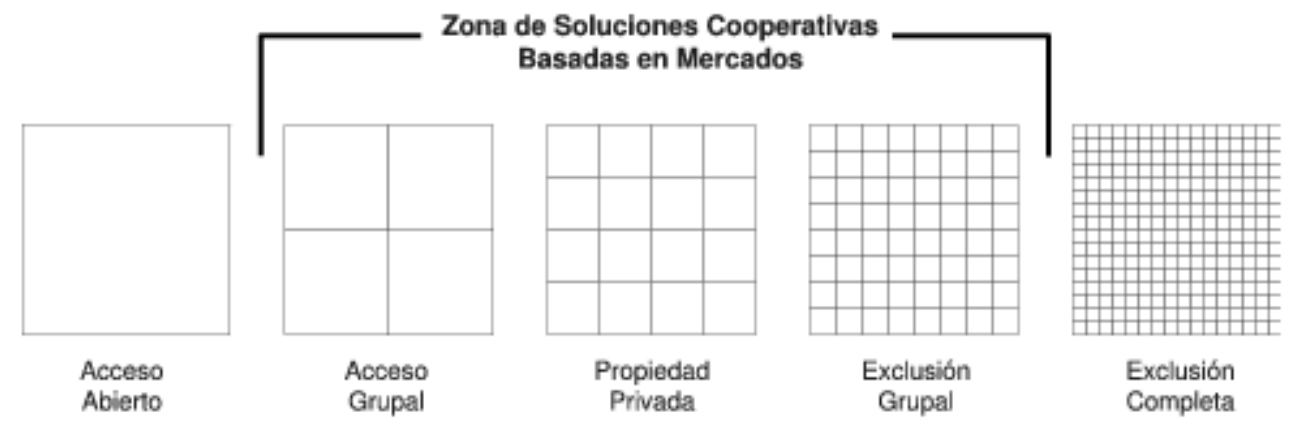

Nos encontramos, entonces, ante tragedias simétricas tanto en el supuesto de tragedia de los comunes como de anticomunes. En el primer supuesto, la ausencia de derechos de propiedad completos lleva a que cada agente económico decida la intensidad de uso del recurso en base a su beneficio e ignore, entonces, que cada uso adicional que él hace impone un costo al resto de los usuarios imponiéndoles una externalidad negativa que implica una utilización ineficiente del recurso. Del mismo modo, en el segundo supuesto, cada agente económico decide el precio que cobrará por el acceso al recurso en base a su propio beneficio ignorando que cada subida de precio o barrera de acceso que imponga genera un costo para el resto de los cotitulares bajo la forma de una externalidad negativa que induce un uso ineficiente del recurso. Es solo cuando se otorgan derechos de propiedad completos (tanto de uso como exclusión) cuando se internalizan todas las externalidades y el recurso se explota de manera eficiente ${ }^{173}$.

\section{El tratamiento de la propiedad intelectual}

Lo expuesto anteriormente los brinda un panorama general que permite plantearnos si la asignación de titularidades hecha por el sistema jurídico resulta eficiente en el caso de los bienes materia de propiedad intelectual. Sobre la base de sus características, debemos plantearnos si el tratamiento actual es el correcto o, si por el contrario, genera una serie de ineficiencias que deben llevar a repensar un sistema más adecuado.

La propiedad intelectual tiene como piedra angular y bien materia de protección a la información. Un rasgo característico de ésta es la ausencia de rivalidad en el consumo. En efecto, uno puede leer una obra de Cervantes, y al mismo tiempo, miles de personas pueden estar leyéndola al mismo tiempo (teniendo en cuenta, por supuesto, que nos referimos a la obra en sí y no al soporte que la contiene). Uno puede ver una película en televisión y muchas personas pueden estar viéndola al mismo tiempo, sin que ello agote el bien. Sobre la información contenida en una obra o en una invención, la que le da forma y valor, no existe rivalidad en el consumo. Más aún, pretender excluir a los demás es costoso, por los que los costos de exclusión altos. El resultado final es que el uso de la propiedad intelectual no

172 CAstillo, Carlos, La economía atascada. En: Manzana Mecánica [blog]. Post del 11 de noviembre de 2011. Disponible en formato electrónico en [http://manzanamecanica.org/2009/11/la_economia_atascada.html]. 
excluye el uso de otros, y, por otro lado, es costoso poner en vigencia mecanismos para que dicha exclusión llegue a funcionar.

Imaginemos que el soporte es irrelevante. En realidad, no hace falta demasiada imaginación para concebir tal situación: la popularización de Internet y el auge de las nuevas tecnologías han permitido que el soporte pierda importancia. Los contenidos digitales alojados en la red, por un lado, y el abaratamiento del costo marginal de producción de soportes (discos duros, memorias, etc.) de alta capacidad son solo una muestra de ello. Pongamos como ejemplo un artículo en un blog, los planos, procedimientos y métodos de fabricación de una invención almacenados en una "nube" o un ensayo en una página web. Millones de personas pueden tener acceso simultáneo a la información en cuestión, sin que el consumo de alguna de ellas sea rival para las demás. Así, la ausencia de rivalidad en el consumo se hace muchísimo más evidente.

El costo de excluir a otros de una obra resulta altísimo. Las personas pueden tener acceso a la información contenida en un artículo, y puede guardarla, copiarla o incluso publicarla en la red. Los costos de desarrollar técnicas de encriptación y de sistemas anti copia son tan elevados, que requieren inversiones de capital de bastante magnitud, sin por ello obtener hasta el momento un resultado eficiente. Por otro lado, el desarrollo de las nuevas tecnologías ha permitido sistemas de copia más eficientes, elevando aún más los costos de exclusión.

Lo mismo va a ocurrir en el caso de las patentes. Si invento una fórmula para un medicamento que cure la enfermedad de Parkinson, dicha fórmula podrá ser utilizada por varios laboratorios al mismo tiempo para la fabricación del medicamento, sin que dicho uso interfiera con la posibilidad de que otro la utilice de manera simultánea. Una vez que la fórmula sea de conocimiento público, los costos de excluir a los otros laboratorios de la producción del medicamento serían realmente prohibitivos, cuando no imposibles de asumir.

En ambos casos, la exclusión de un tercero puede a su vez generar elevados costos terciarios (es decir, el costo de los procesos legales), que pueden ser realmente enormes. Esto no se presenta en el caso de bienes materiales. Al respecto, señala PASQUEL:

"Una patente o los derechos de autor sobre una famosa obra pueden ser una mina de oro. El oro, sin embargo, tiene consumo rival y bajos costos de exclusión, por lo que basta tenerlo bien guardado para que otros no se aprovechen del mismo. Ello no sucede con la información protegida por la patente o los derechos de autor. Ésta es relativamente fácil de copiar y aprovechar. Ello genera que numerosas empresas e individuos utilicen la información violando el derecho de propiedad del titular. Éste, a su vez, tendrá que iniciar acciones legales para impedir el uso ilícito.

Los costos de exclusión de los bienes materiales no suelen suponer más que guardar el bien en un sitio seguro o contratar un buen grupo de guardias que lo vigilen. En el caso de la información, los costos de exclusión suponen inversiones que muchas veces ascienden a millones de dólares en procesos legales. ${ }^{174}$ 
La rivalidad en el consumo es una consecuencia de la escasez de los bienes. Si no existe tal escasez, el consumo de los bienes no es rival, pues hay bienes suficientes para todos. En ese sentido, el símil del Jardín del Edén de Kinsella cobra sentido. Si estuviéramos en un lugar donde la tierra y los bienes fueran infinitos, no tendría sentido la formulación de reglas de propiedad.

"Were we in a Garden of Eden where land and other goods were infinitely abundant, there would be no scarcity and, therefore, no need for property rules; property concepts would be meaningless. The idea of conflict, and the idea of rights, would not even arise. For example, your taking my lawnmower would not really deprive me of it if I could conjure up another in the blink of an eye. Lawnmower-taking in these circumstances would not be "theft." Property rights are not applicable to things of infinite abundance, because there cannot be conflict over such things. Thus, property rights must have objective, discernible borders, and must be allocated in accordance with the first occupier homesteading rule. Moreover, property rights can apply only to scarce resources". ${ }^{175}$

En efecto, como sugiere KINSELLA, la propiedad no se aplica a cosas infinitamente abundantes porque no surge conflicto en su uso, al no existir escasez de los mismos. Si un bien puede reproducirse rápidamente, se comporta como la podadora mágica del ejemplo, que se reproduce con un simple toque de la mano. En ese contexto, la exclusividad resulta innecesaria.

Es justamente esa facilidad de copia y reproducción la que hace que bienes como aquellos que son objeto de propiedad intelectual no sean escasos. Si escribo un libro y otro lo copia, aún poseo el libro y el soporte, pero quien lo copia tiene el patrón de palabras que le va a permitir "ensamblar" e imprimir su propio libro. En ese sentido, el uso no excluye el uso de otros, por lo que no se presenta rivalidad en el consumo, al no haber escasez. Sin escasez no existe la posibilidad de conflictos de uso sobre un bien escaso, que es la base y el fundamento de los derechos de propiedad privada.

"The problem with IP rights is that the ideal objects protected by IP rights are not scarce; and, further, that such property rights are not, and cannot be, allocated in accordance with the first occupier homesteading rule, as will be seen below. Scarcity and Ideas Like the magically-reproducible lawnmower, ideas are not scarce. If I invent a technique for harvesting cotton, your harvesting cotton in this way would not take away the technique from me. I still have my technique (as well as my cotton). Your use does not exclude my use; we could both use my technique to harvest cotton. There is no economic scarcity and no possibility of conflict over the use of a scarce resource. Thus, there is no need for exclusivity. Similarly, if you copy a book I have written, I still have the original (tangible) book, and I also still "have" the pattern of words that constitute the book. Thus, authored works are not scarce in the same sense that a piece of land or a car are scarce. If you take my car, I no longer have it. But if you "take" a book-pattern and use it to make your own physical book, I still have my own copy". 176

Teniendo en cuenta lo anterior, nos encontramos con bienes que no tienen rivalidad en el consumo y con altos costos de exclusión. Como mencionamos anteriormente, si identificamos un bien sin rivalidad en el consumo y con altos costos de exclusión, el uso

175 Kinsella, N. Stephan. Against Intellectual Property [en línea]. En: Journal of Libertarian Studies. 2001. Vol. 15, No 2. Disponible en Internet en: <www.mises.org> Ídem. 
público parece la alternativa más eficiente. Puede pensarse que en el beneficio social que se lograría si las obras de Mario Vargas Llosa o Gabriel García Márquez estuvieran en el dominio público y fueran libremente accesibles. Muchísima gente se vería beneficiada si pudiera ser curada con medicamentos fabricados por un sinnúmero de laboratorios al mismo tiempo, lo que reduciría su costo final. Y lo mejor de todo, sin impedir al autor o al inventor usar también su obra o invento.

Por otra parte, los costos de exclusión en la propiedad intelectual suelen ser muy altos, debido a su carácter incorporal. Los bienes físicos son más fáciles de defender: basta construir una cerca para proteger una casa, contratar unos vigilantes para cuidar un banco, o mantener guardado un ordenador para evitar que lo use otro. Sin embargo, basta memorizar un poema para recitarlo, bajar una canción de un servidor $\mathrm{p} 2 \mathrm{p}$ para reproducirlo en cualquier momento y copiar un archivo epub a un smartphone o tablet para leerlo en cualquier momento. Al tratarse de un derecho que puede ser reproducido ad infinitum, el control sobre el mismo es realmente costoso.

\section{6. ¿Qué papel debe jugar la propiedad intelectual?}

Como hemos podido apreciar en el análisis anterior, la propiedad intelectual carece de rivalidad en el consumo y sus costos de exclusión son elevados, lo cual es exactamente lo inverso a lo que encontramos en la propiedad privada clásica. Normalmente, en estos casos, el sistema jurídico asigna a estos bienes la consideración de bienes públicos, al carecer de los atributos clásicos de la propiedad.

Sin embargo, en el caso de la propiedad intelectual, el sistema jurídico decide protegerla otorgando derechos de exclusiva, un monopolio temporal, creando así una suerte de "propiedad artificial”. ¿Por qué? ¿Es que acaso se persigue que cumpla una función económica que justifique esto?

La respuesta que se da tradicionalmente a esta cuestión es que la función de la propiedad intelectual es la de incentivar la creatividad y la innovación. Es ésta la razón para crear un sistema artificial capaz de producir incentivos, para que las personas inviertan tiempo y recursos en generar creaciones útiles para todos, aun cuando para ello tengamos que ir contra la lógica económica de la que deriva la asignación de titularidades como el derecho de propiedad.

Se considera que sin los incentivos creados por la propiedad intelectual, las personas no invertirían tiempo y recursos en la creación. De esta manera, para estimular la creatividad de los autores, se crea un monopolio temporal artificial sobre la obra por un periodo de tiempo determinado.

Para incentivar la creatividad de los autores e inventores, se fuerza un derecho de exclusiva, costoso de ejecutar y difícil de poner en práctica. De esta forma, se otorga a Apple por veinte años la patente sobre los dispositivos electrónicos rectangulares con esquinas redondeadas $^{177}$, para motivarla a seguir innovando, y se conceden derechos de autor a Joaquín Sabina hasta setenta años después de su muerte para motivarlo a componer música. Por ello, asumimos los inmensos costos de poner en vigencia este tipo de derechos y

177 El documento con la patente en cuestión puede ser obtenido en su totalidad, en formato PDF, en la URL <http://assets.sbnation.com/assets/1701443/USD670286S1.pdf>. 
aceptamos que, al menos por un tiempo, estos derechos sean privados (creando un monopolio o privilegio) a pesar que su mejor uso, desde el punto de visto social, es el uso público ${ }^{178}$.

Los costes del sistema y la creación de una "escasez artificial" para crear un monopolio en favor de los autores para incentivar la creación explican por qué tenemos que sujetar este tipo de derechos a normas distintas a las del Libro de Derechos Reales del Código Civil. Pero también explica por qué este tipo de derechos no pueden ser perpetuos, a diferencia de la propiedad clásica.

La idea no es proteger en sí mismo un bien sin rivalidad en el consumo, sino los incentivos para crear. En tal sentido, no es necesario otorgarle al creador un monopolio o privilegio perpetuo sobre su idea, sino que basta con otorgarle los incentivos para crear. Vencido el plazo, el bien pasa a dominio público, donde conseguirá el uso más deseable desde el punto de vista social-económico, es decir, el uso libre por todos.

El problema que se presenta con el actual sistema de propiedad intelectual es que no ha sabido encontrar el equilibrio adecuado para promover la creatividad sin convertirse en un costo excesivo para los consumidores de los bienes producidos. Lo que debe buscar un sistema eficiente de propiedad intelectual es incentivar la actividad creativa de los autores, de manera que compense el coste de oportunidad de dedicarse a cualquier otra actividad que no sea la creativa.

En el sentido de lo señalado, la función económica de los sistemas de propiedad intelectual es incentivar la actividad creativa compensando el costo de oportunidad del creador sin convertirse en un costo social excesivo:

"La eficiencia requiere que, en caso de que el innovador se decida por la innovación, reciba de esta última al menos lo que hubiera recibido de la actividad alternativa, esto es: su costo de oportunidad. Cuando la estructura del mercado permite al innovador recibir más que su costo de oportunidad, esta renta adicional no tiene ninguna utilidad social. Per se, esta renta adicional podría ser una simple transferencia que no afecta la eficiencia económica; sin embargo, más frecuentemente que no, y en particular cuando está involucrado algún poder monopólico, esta renta adicional se le debe al innovador porque tiene incentivos a proveer menos innovaciones, o menos copias de sus innovaciones, que lo socialmente eficiente. En este caso, la renta adicional no es únicamente una transferencia neutral de los consumidores a los innovadores (lo que podría ser injusto, pero irrelevante para la eficiencia) sino un impuesto socialmente costoso e ineficiente a los consumidores, menos copias disponibles para la gente que lo deseable y factible tecnológicamente". ${ }^{179}$

Pero ello no ocurre así. Como veremos en los siguientes artículos, el actual sistema actual de propiedad intelectual no resulta eficiente al no haber encontrado el equilibrio entre la renta destinada a compensar el costo de oportunidad de los creadores e innovadores y el costo social del sistema. Por el contrario, sucesivas extensiones de la duración de los derechos de autor, así como una aplicación más restrictiva de los mismos, ha devenido en un sistema que no incentiva la creación, sino que crea autores - rentistas, que preocupados por la

178 BULLARD, Alfredo. Óp. cit. pp. 209-210.

179 BOLDRIN, Michele; LEVINE, David, Propiedad intelectual y asignación eficiente del beneficio social de las innovaciones [en línea]. En: Cuadernos Económicos del ICE, No 76, 2008, pp. 96-97. Documento en Internet en: <http://www.revistasice.com> 
pérdida de sus privilegios, forman grupos de presión que abogan por legislaciones cada vez más restrictivas, aun cuando en el camino se violen derechos de los individuos.

Por su parte, al relajarse los requisitos necesarios para obtener una patente, se conceden una serie de titularidades sobre información que no debiera tenerlas, al no tener el nivel inventivo requerido, no ser novedosas o carecer de aplicación industrial ${ }^{180}$. Este exceso de titularidades no solo no incentiva la innovación, sino que paradójicamente ralentiza, dificulta o incluso paraliza el proceso inventivo, produciéndose un problema de anticomunes.

En la siguiente entrega, veremos que existen alternativas más eficientes, que van desde la reducción de los actuales niveles de protección otorgados por el sistema actual, hasta la posible eliminación de la propiedad intelectual como regla general a favor de un sistema de mercado competitivo basado en nuevos modelos de negocio.

180 Un claro ejemplo de esto es la patente concedida en 2001 por la Oficina Australiana de Patentes en favor de John Michael Kheog por un "dispositivo circular para facilitar el transporte". Este dispositivo existe desde hace más de 5000 años, y es más conocido como "rueda". El documento que contiene la referida patente puede ser consultado en formato PDF en <http://www.hl7.org.au/docs/Australian\%20Patent\%202001100012.pdf〉. 